\section{Wolfgang Clausen}

\section{Die Staatwerdung Ghanas}

Studie über die Verfassungsentwicklung kolonialer Gebiete zum unabhängigen Staat

Franz Appel Verlag, Hamburg 1966

(Veröffentlichungen des Instituts für Internationales Recht an der Universität Kiel, Band 53)

XII, 196 S., DM 28,-

Die Entstehung der neuen Staaten in Asien und Afrika dürfte auf lange Sicht gesehen eines der bedeutendsten Ereignisse unseres Jahrhunderts sein, und zwar nicht nur für die Politik, sondern auch für die Staatslehre, die hier in seltener Weise an einer sehr schnell ablaufenden Entwicklung ihre Thesen überprüfen und gegebenenfalls vertiefen kann. Untersuchungen über die Staatwerdung asiatischer und afrikanischer Staaten dürfen daher immer mit einem großen Interesse rechnen.

Clausen hat es unternommen, die Staatwerdung Ghanas, insbesondere die Verfassungsentwicklung darzustellen. Dies ist in mehrfacher Hinsicht ein reizvolles Unternehmen. Ghana war der erste Staat, der in Afrika südlich der Sahara die Unabhängigkeit erhielt, und die Goldküste, wie Ghana zur Zeit der britischen Kolonialherrschaft hieß, war die westafrikanische Besitzung Großbritanniens, die sowohl in ihrer inneren Entwicklung als auch in der Ausbildung der Bevölkerung am weitesten fortgeschritten war. Die Goldküste war damit auch die britische Kolonie in Afrika, die dem Mutterland durch eigene Wünsche und Vorstellungen von der Zukunft zunächst die größten Schwierigkeiten bereitet hat und die im Vergleich etwa zu den ostafrikanischen Besitzungen besonders eigenwillig und lebendig war. Im Gebiet der Goldküste gab es außerdem schon vor der britischen Besitznahme afrikanische Herrschaftssysteme, die sich über größere Territorien erstreckten und eine eigenständige stabile Ordnung hatten. Die Goldküste war auch der Teil Afrikas, der sehr früh schon Kontakt zu Europa hatte und dessen Einwohner im Rahmen dieser Beziehungen zu Europa sehr früh europäisches Den- ken angenommen hatten und, wie man in Ghana heute noch mit Stolz bemerkt, in früheren Jahrhunderten als Dichter oder Gelehrte in Europa zu Ruhm gekommen waren. In Ghana bestanden also für afrikanische Verhältnisse die denkbar besten Voraussetzungen, einen unabhängigen Staat aufzubauen. Das bedeutet aber auch, daß Probleme, die bei der Staatwerdung Ghanas auftreten, für andere neue afrikanische Staaten in noch sehr viel größerem Umfang zu beobachten sein werden. Eine Untersuchung über Ghana verdient daher größte Beachtung.

Clausen geht an sein Thema von den Texten der Verfassungen der Goldküste und Ghanas heran. Er zeichnet an Hand der englischen Publikationen die Stufen der Entwicklung von der Goldcoast-Kolonie über die Eroberung der Ashanti-Konföderation, das sogenannte Protektorat über die Nordgebiete bis zu der Úbernahme des Mandats über Teile von Deutsch-Togo nach. Er stellt ausführlich alle Ordnungen dar, die zu irgendeiner Zeit unter britischer Herrschaft im Bereich der Goldküste gegolten haben. Die Untersuchung gibt damit erstmalig im deutschen Sprachbereich eine zusammenfassende Darstellung der Verfassungsgeschichte Ghanas. Jedoch die Staatwerdung Ghanas wird dadurch in einen sehr formalen Rahmen gebracht, der Wesen und Problematik der Staatlichkeit in den neuen Staaten Afrikas und Asiens überhaupt nicht in Erscheinung treten läßt. Diese Kritik trifft allerdings nicht den Verfasser allein, sondern sie ist bedauerlicherweise bei vielen deutschen Untersuchungen über die neuen Staaten angebracht; es fehlt an einer Beziehung zur Wirklichkeit, so $\mathrm{da}$ die Untersuchung weitgehend kompilatorischen Charakter trägt. Aus Werken von Lokalkennern werden die juristischen Tatbestände zusammengetragen, ohne daß deren Bedeutung in der Wirklichkeit erfaßt und dargestellt wird. Die Ursache dieses Úbelstandes ist nicht so sehr der böse Wille oder die Unfähigkeit der Autoren, sondern einfach die in Deutschland weit verbreitete Vorstellung, daß wissenschaftliche Aus- 
landskunde besonders bei nichteuropäischen Staaten ohne Kenntnis der lokalen Verhältnisse, oder, wie man es neuerdings nennt, ohne regionalwissenschaftliche Grundlegung betrieben werden kann. Das führt dann dazu, daß praktisch nur der Text einer Verfassung referiert wird, wobei Eigenheiten, die vom europäischen Modell abweichen, recht verständnislos angemerkt werden und häufig gar nicht in einen richtigen Zusammenhang kommen.

Clausen versucht die Gesamtverfassung der Kolonien und des Staates darzustellen, einschließlich der Lokalverwaltung. Bereits in der Einleitung hat er darauf hingewiesen, daß er gerade auf die untere Ebene der Lokalverwaltung Wert legt, aber bei der Darstellung dieser Ebene zeigt sich auch am deutlichsten die Schwäche der Arbeit. Clausen weiß zwar, daß Ghana die Größe der britischen Inseln, aber heute nur etwa 6 Millionen Einwohner hat. Um 1900, als die Kolonie ihre letzte Form erhielt, dürften es noch weit weniger Einwohner gewesen sein. Daraus ergibt sich, daß die Besiedlungsdichte Ghanas mit der Besiedlungsdichte Europas im Frühmittelalter verglichen werden und daß die lokale Verwaltung diesen Verhältnissen gemäß mehr feudalistische als staatliche Züge tragen muß. Unter derartigen Bedingungen ist es so gut wie unmöglich, lokale Verwaltungen einzurichten, die europäischen Maßstäben gerecht werden. Das System der „indirect rule", das von Großbritannien bereits vor Lugard praktiziert wurde, lief darauf hinaus, bestehende lokale Machthaber in die Kolonialverwaltung einzugliedern und sie unter Umständen sogar in ein Vasallenverhältnis zur britischen Krone zu bringen. Diese lokalen Machthaber wurden zwar zur Durchsetzung notwendiger Maßnahmen angehalten, in der Regel überließ man aber die Ordnung der örtlichen Verhältnisse ihrem Belieben, da bei der geringen Effektivität, die die Kolonialverwaltung hatte, es unmöglich war, Anordnungen für lokale Reformen wirklich durchzusetzen. Anders war es nur in den wenigen Städten. Clausen, der diese Zusammenhänge außer acht läßt, wundert sich, daß in den Städten schon sehr früh eine echte Selbstverwaltung durch die Afrikaner eingeführt wurde und beklagt ein wenig die mangelnde Demokratisierung in den weiten ländlichen Bezirken.

Schon bei der Diskussion um die Verfassung des unabhängigen Staates wurde dieses Problem deutlich in dem Streben der Convention Peoples Party, eine zentrale Staatsgewalt einzurichten, die durch Mobilisierung der Massen in der Lage war, endlich eine Modernisierung der Gesellschaft durchzusetzen. Die CPP warf daher Großbritannien vor, Ghana bewußt nicht entwickelt, sondern durch Zusammenarbeit mit den reaktionären Häuptlingen in den Landgebieten rückständig gehalten $\mathrm{zu}$ haben - ein Vorwurf den Clausen zwischen den Zeilen wiederholt. Die CPP wollte es besser machen. Mit kühnen Verfassungsänderungen, die Clausen ausführlich darstellt, entmachteten sie die Häuptlinge vollkommen; aber es gelang ihr dennoch nicht, in dem weiten Land mit den wenigen Menschen eine effektive innere Ordnung herzustellen. Nach vergeblichen Bemühungen wandte sich Nkrumah sehr schnell der Außenpolitik $\mathrm{zu}$, in der Hoffnung, hier ein geeignetes Betätigungsfeld $\mathrm{zu}$ finden. Und als er gestürzt wurde, wurde ihm gerade zum Vorwurf gemacht, die innere Entwicklung nicht vorangetrieben zu haben. Er ist unter anderem an der Aufgabe gescheitert, die Großbritannien gar nicht erst angegangen hat. Er hat zwar die Häuptlinge entmachtet und wenn sie Widerstand leisteten, rücksichtslos beseitigt; er hat auch aus den Reihen seiner Partei "moderne“ lokale Verwalter eingesetzt; aber ihre Isolierung von der fernen Staatsgewalt in Accra und den Provinzhauptstädten war bei der geringen Erschließung des Landes so groß, daß sie von Vertretern der Staatsgewalt sehr schnell wieder zu Herren der Kommunen wurden, d. h. zu Häuptlingen neuer Art, mit eigenen und durch mangelnde Bindung an Stammestraditionen oft sehr eigennützigen Interessen. Auch die Diktatur Nkrumahs war nicht in der Lage Abhilfe zu schaffen, zumal Nkrumah selbst kein 
Vorbild an Uneigennützigkeit gewesen ist und sich mehr als Herrn und weniger als Diener des Staatswesens betrachtet hat. Die Armee, die auf Grund einer gediegenen englischen Ausbildung bis in die jüngste Zeit klarere Vorstellungen von staatlicher Ordnung hat als Nkrumahs Gruppe, hat die Diktatur Nkrumahs beseitigt; ob sie allerdings eine moderne Ordnung für den Staat Ghana schaffen wird, ist noch ungewiß. Eine eingehende Untersuchung dieser Fragen fehlt bei Clausen leider. Wenn man auch in einem im Jahre 1966 gedruckten Buch die Behandlung der allerjüngsten Entwicklung nicht erwarten darf, so ist dennoch zu beanstanden, daß zwei und drei Jahre zurückliegende Ereignisse fehlen. So zum Beispiel das Gesetz zur Demokratisierung der Kommunalverwaltung von 1963, mit dem Nkrumah zum letzen Male versuchte, die lokale Verwaltung fest in die Hand zu bekommen. Weiter fehlt die Verfassungsänderung von 1964, mit der die CPP zur Einheitspartei wurde. Diese Lücken wiegen gering gegenüber dem Hauptmangel der Arbeit, nur eine juristische Textkompilation zu sein. Der Vorzug der Arbeit ist es, erstmalig in deutscher Sprache die Formen der Verfassungsentwicklung von der Goldküste zum Staate Ghana zusammengefaßt dargestellt und erläutert zu haben, was bei der oft schlechten Zugänglichkeit der Texte gewiß verdienstlich ist. Ob allerdings derartige Arbeiten ausreichen, die deutsche wissenschaftliche Auslandskunde im internationalen Maßstab beachtenswert $\mathrm{zu}$ machen, muß angesichts vorzüglicher amerikanischer, englischer, französischer aber auch belgischer Untersuchungen bezweifelt werden.

Dr. Dieter Schröder, Lübeck

\section{E. I. Nwogugu}

The Legal Problems of Foreign Investment in Developing Countries

Manchester University Press, Manchester 1965, 320 S., sh 45

Diese in ursprünglicher Form als Dissertation an der Universität Manchester eingereichte Arbeit beleuchtet die rechtlichen Aspekte der mit privaten Investitionen in Entwicklungsländern verbundenen Fragen. Das vorliegende Buch verdient nicht nur hinsichtlich seiner Aktualität, sondern auch - und vor allem - deshalb Beachtung, als hier erstmals durch einen Juristen aus einem jungen afrikanischen Staat (Nigeria) der Versuch gemacht wird, die Investionsproblematik in ihrem vollem Umfang zu erörtern.

Im ersten Teil seiner Arbeit (S. 9-30) geht Nwogugu auf investitionshemmende rechtliche Maßnahmen sowohl kapitalimportierender als auch kapitalexportierender Staaten ein. Zur ersten Gruppe zählt er Investitionskontrollgesetze, die z. B. eine Zwangsbeteiligung von Inländern am Unternehmen oder die Verpflichtung zur Verwendung von inländischen Arbeitskräften usw. vorsehen, ferner diskriminierende Steuergesetzgebung und als Haupthindernis die Furcht vor Nationalisierung oder entschädigungsloser Enteignung; doch nimmt der Verfasser im Anschluß an Fachiri, Cheng, Hyde und Wortley eine Entschädigungspflicht des enteignenden Staates als Norm des Völkergewohnheitsrechtes an (S. 22, 23 und 119). Von der Seite der kapitalexportierenden Staaten her stellen Doppelbesteuerung und Monopolgesetzgebung die hauptsächlichsten investitionshemmenden Maßnahmen dar.

Kernstück der Arbeit bildet Teil II (S. 33-225), der sich mit den rechtlichen Methoden zur Förderung und zum Schutz von privaten Investitionen befaßt. Steuer- und Zollbegünstigungen, die Beseitigung von Devisenverkehrsbeschränkungen zur Ermöglichung des Rücktransfers von Gewinnen aus Kapitalanlagen sowie Abkommen zur Vermeidung von Doppelbesteuerungen sind nach Ansicht des Verfassers geeignete Maßnahmen zur Förderung verstärkter internationaler Investitionstätigkeit. Mit Recht kritisiert der Autor jedoch die von einer Reihe von sogenannten Entwicklungsländern ausländischen Investoren zugesicherte Freiheit von Einkommen- oder Gewinnsteuern für die ersten fünf Jahre der Tätigkeit, da in 